\title{
Observation of shocks associated with CMEs in 2007
}

\author{
H. Aryan ${ }^{1}$, M. A. Balikhin ${ }^{1}$, A. Taktakishvili ${ }^{2}$, and T. L. Zhang ${ }^{3}$ \\ ${ }^{1}$ Department of Automatic Control and Systems Engineering, University of Sheffield, Sheffield, UK \\ ${ }^{2}$ NASA Goddard Space Flight Center, Greenbelt, Maryland, USA \\ ${ }^{3}$ Space Research Institute, Austrian Academy of Sciences, Graz, Austria \\ Correspondence to: H. Aryan (aryan.homayon@gmail.com)
}

Received: 29 March 2013 - Revised: 11 November 2013 - Accepted: 6 February 2014 - Published: 12 March 2014

\begin{abstract}
The interaction of CMEs with the solar wind can lead to the formation of interplanetary shocks. Ions accelerated at these shocks contribute to the solar energetic protons observed in the vicinity of the Earth. Recently a joint analysis of Venus Express (VEX) and STEREO data by Russell et al. (2009) have shown that the formation of strong shocks associated with Co-rotating Interaction Regions (CIRs) takes place between the orbits of Venus and the Earth as a result of coalescence of weaker shocks formed earlier. The present study uses VEX and Advanced Composition Explorer (ACE) data in order to analyse shocks associated with CMEs that erupted on 29 and 30 July 2007 during the solar wind conjunction period between Venus and the Earth. For these particular cases it is shown that the above scenario of shock formation proposed for CIRs also takes place for CMEs. Contradiction with shock formation resulting from MHD modelling is explained by inability of classical MHD to account for the role of wave dispersion in the formation of the shock.
\end{abstract}

Keywords. Interplanetary physics (solar wind plasma) ionosphere (modeling and forecasting; particle acceleration)

\section{Introduction}

Coronal Mass Ejection (CME) is a sudden eruption of plasma particles from the Sun (e.g., Jian et al., 2006; Kahler et al., 1988). CMEs mainly erupt from the active regions of the Sun's surface and can travel with speeds of hundreds to 23 thousands $\mathrm{km} \mathrm{s}^{-1}$ carrying large quantities of coronal material (Hundhausen, 1999). The plasma released consists of energetic electrons and protons, and may also contain other elements such as, iron, oxygen and helium (Gosling et al., 1993). CMEs are released as a result of huge disturbances and changes in the Sun's coronal magnetic field and are usually observed using white-light coronagraph (Jian et al., 2006). CME eruption is common during the solar maxima with up to five CMEs per day, but less common during the solar minima where on average only one CME eruption is expected per day (Jian et al., 2006). Fortunately, only the halo and partial halo CMEs travel towards the Earth (Gopalswamy et al., 2005). However, all CMEs undergo an effective acceleration due to interaction with the solar wind (Gopalswamy et al., 2000) as they propagate; slow moving CMEs are accelerated while fast moving CMEs are decelerated towards the speed of solar wind (Gosling et al., 1993). The interaction of the CMEs with the solar wind may lead to the formation of interplanetary collisionless shocks and also contribute to the acceleration of solar energetic protons in both the vicinity of the Earth and the interplanetary medium. Fermi type ion acceleration at the collisionless shocks takes place due to the multiple crossings of shocks by a particular ion. Initially a seed population of the reflected ions is formed which excites wave instabilities upstream of the shock front. Interaction of the reflected ions with formed upstream wave field leads to the ion scattering. Part of the reflected ions are scattered back to the shock front that can be reflected back again and so on. Each time a particular ion is reflected from the shock front it gains energy. While such a general scenario is widely accepted a few unsolved problems still remain, for example, the origin of the seed population and a comprehensive self-consistent model of plasma wave interactions and ion scattering in the wave field. Currently, magnetohydrodynamics (MHD) codes are used to model the formation and propagation of shocks associated with CMEs. Forecasting solar energetic protons on the basis of these MHD models require waves and ion seed populations to be added to the field structure obtained by the MHD codes. This cannot provide 


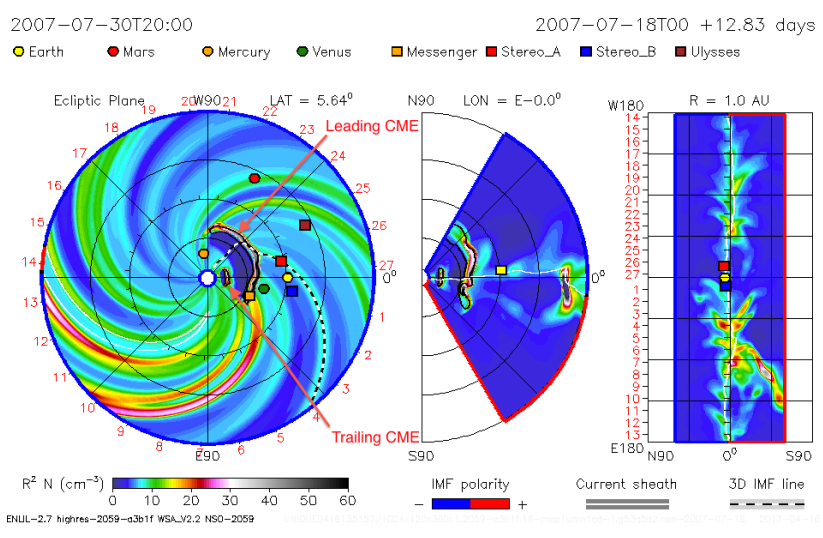

Fig. 1. The CCMC's ENLIL with a cone model simulation results. The estimated arrival time of the leading partial halo CME at Venus is approximately 20:00 on 30 July 2007 using the above simulation results. The red arrows mark the leading and trailing CMEs.

accurate results as the process of acceleration is sensitive to both the peculiarities of the seed population and the distribution of wave amplitudes, that cannot be obtained by the MHD approximation. In the present study it is shown that at least for some particular CMEs the MHD approach is unable to account even for the formation and evolution of a CME associated shock. One of the main motivation for the present study is the results of Russell et al. (2009) showing that for CIR associated shocks it is the region between Venus and the Earth where stronger shocks are formed as a result of coalescence of weaker shocks and Russell et al. (2009) even refers to that region as the incubator of shocks. In the present study it is shown that this is also the case at least for some CMEs.

\section{Data}

The magnetic field data used to identify shock crossings at the Venusian orbit $(0.72 \mathrm{AU})$ were recorded by the fluxgate magnetometer on board VEX spacecraft (Zhang et al., 2006). VEX was launched by the European Space Agency (ESA) using a Soyuz-Freget launcher in November 2005 carrying a number of different instruments to study Venus and it has been in operation since April 2006. Whereas the magnetic field data used to identify shock crossings at the Earth orbit (1 AU) were recorded by the magnetometer on board ACE spacecraft. ACE was launched by the Office of Space Mission and Payload Development Division of the National Aeronautics and Space Administration (NASA) using a McDonnell-Dougles Delta II 7920 launch vehicle in August 1997 carrying a number of different sensors and instruments to measure and compare the composition of several samples of matter (Stone et al., 1998). The CME parameters, such as eruption time, direction of propagation, speed, acceleration, cloud mass, kinetic energy and angular width were obtained from the Large Angle and Spectrometric Coronagraph

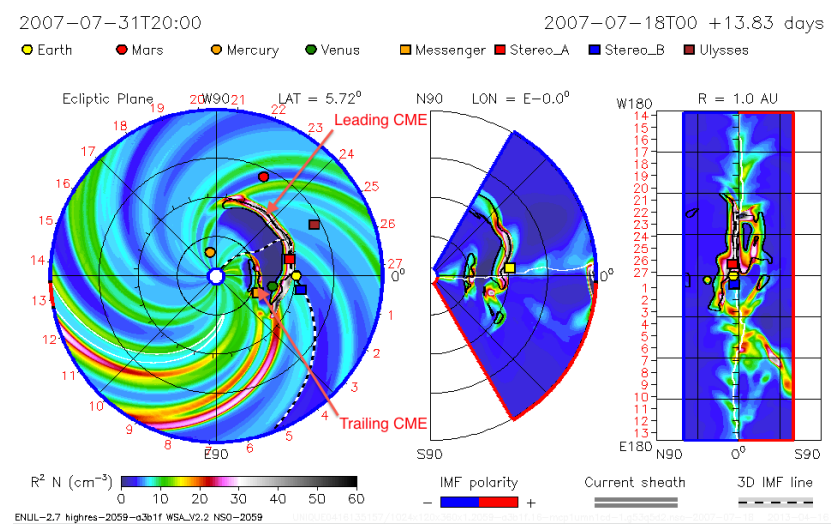

Fig. 2. The CCMC's ENLIL with a cone model simulation results. The estimated arrival time of the leading partial halo CME at the Earth is approximately 20:00 on 31 July 2007 using the above simulation results. The red arrows mark the leading and trailing CMEs.

Experiment (LASCO) CME catalogue which is available online (http://cdaw.gsfc.nasa.gov/CME_list/). LASCO is one of several instruments on board the Solar and Heliospheric Observatory (SOHO) spacecraft which was launched using an Atlas II-AS (AC-121) in December 1995 as part of an international collaboration between ESA and NASA to study the Sun. The ENLIL simulation results have been provided by the Community Coordinated Modelling Center (CCMC) at Goddard Space Flight Center through their public Runs on Request system (http://ccmc.gsfc.nasa.gov). The CCMC is a multi-agency partnership between NASA, AFMC, AFOSR, AFRL, AFWA, NOAA, NSF and ONR. The ENLIL with a cone model (Odstrcil et al., 2004) which is a 3-D time dependent MHD solar wind model, capable of representing CMEs and propagating features through a realistic model of the solar wind, was developed by the D. Odstrcil at the University of Colorado at Boulder. The CCMC version of the ENLIL model has been verified against data recorded near Earth showing that the model provides good predictions of the CME arrival times (e.g., Falkenberg et al., 2010, 2011; Taktakishvili et al., 2009).

\section{Event}

This paper studies CME associated shocks during the solar wind conjunction period between Venus and the Earth in 2007. During this period only two CMEs (halo/partial halo) have been observed and recorded by SOHO/LASCO; a partial halo CME eruption at 01:31 UTC on 29 July 2007 followed by a halo CME eruption a day later at 04:54 on 30 July 2007. The relevant input CME parameters required by the ENLIL model to simulate the propagation of the above two CMEs were obtained from the SOHO/LASCO catalog in order to predict the arrival times of the CMEs at the Venusian and the Earth orbits. The simulation results of the ENLIL 

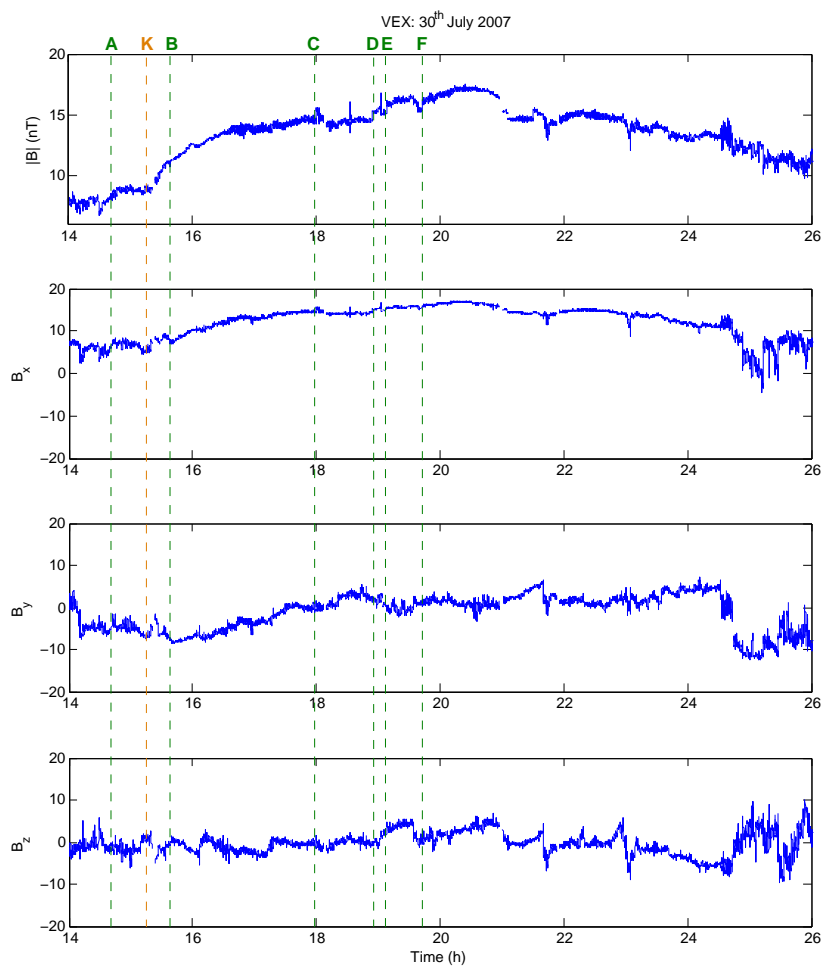

Fig. 3. Magnetic field data recorded by VEX during the CME crossing period on 30 July 2007. The vertical green dotted lines mark the shock crossing times (A:F) and the vertical orange dotted line (K) represent an example of a nonlinear structure that has not fully formed into a shock.

with a cone model are presented as snapshots in Figs. 1 and 2 which were used to estimate the arrival time of the leading CME at VEX and ACE as approximately 20:00 on 30 July 2007 and 20:00 on 31 July 2007 respectively. On the other hand the estimated arrival time of the trailing CME at VEX and ACE is approximately 02:00 on 1 July 2007 and 02:00 on 2 July 2007 respectively. Results from the ENLIL with a cone model provides a time slot to search for possible shocks associated with the crossing of the corresponding CMEs. The main advantage of this method is that it eliminates any ambiguity regarding the CME crossing time. Generally, the majority of CME associated shocks travel ahead of the propagating CME (Jian et al., 2006). Therefore, the CME associated shock crossings would mainly be expected to occur close to the CME arrival time at the Venusian and the Earth orbits.

The magnetic field measurements recorded by VEX and ACE, as presented in Figs. 3 and 4 respectively, show the crossing of the leading partial halo CME at both VEX and ACE. During these periods a number of nonlinear structures reminiscent of shock crossings have been observed both at VEX and ACE. All such structures were analysed in order to identify shock crossings using the same shock crossing criteria as developed by Russell et al. (2009). According to this
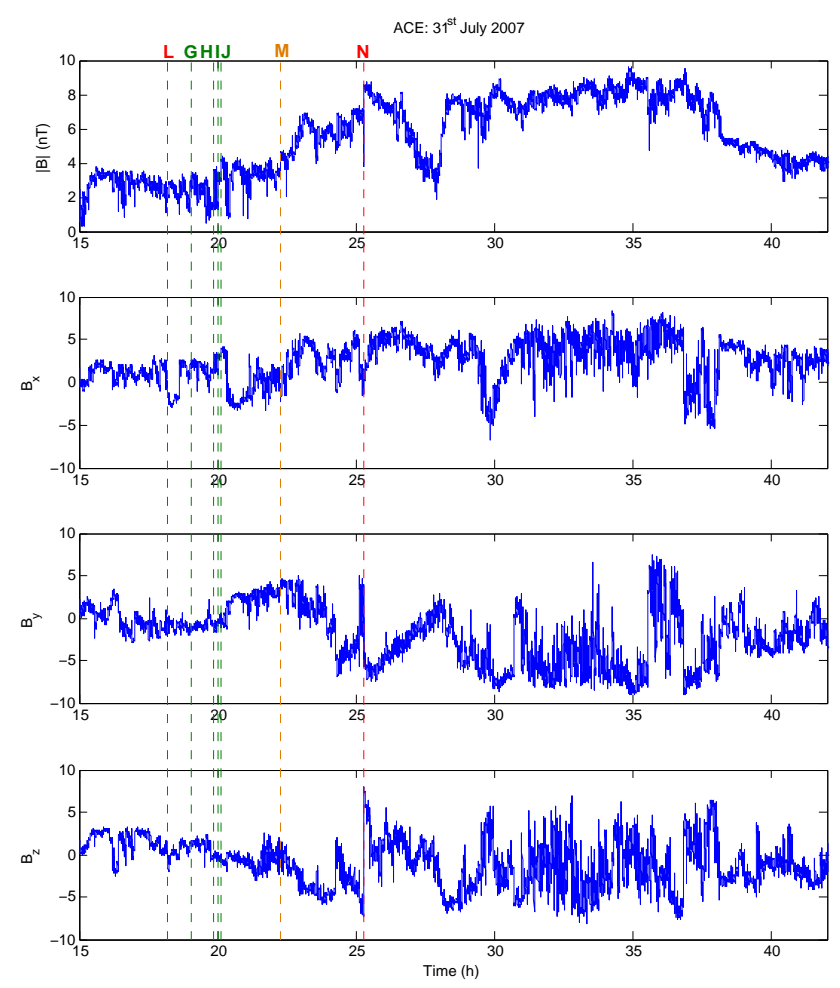

Fig. 4. Magnetic field data recorded by ACE during the CME crossing period on 31 July 2007. The vertical green dotted lines $(\mathrm{G}: \mathrm{J})$ mark the shock crossing times, the vertical orange dotted line (M) represent an example of a nonlinear structure that has not fully formed into a shock and the red dotted lines ( $\mathrm{L}$ and $\mathrm{N}$ ) represent some examples of nonlinear structures that did not fulfil the criteria for a shock crossing.

criteria, for a shock crossing the directions of the shock normal $\left(B_{\mathrm{n}}\right)$, the main magnetic field component and the component in the shock plane parallel to the projection of the upstream field $\left(B_{L}\right)$ should not cross zero in the shock coordinate frame. Also a shock crossing is characterised by a sharp single directional jump in the magnetic field measurements (Russell et al., 2009) with the Mach number crossing one between upstream and downstream of the shock. When the above criteria was implemented to the nonlinear structures reminiscent of shock crossings associated with the leading CME it was found that six structures observed by VEX at times: 14.54, 15.51, 18.00, 18.91, 19.12 and 19.71 UTC on 30 July 2007, and four structures observed by ACE at times: 19.00, 19.89, 20.03 and 20.11 UTC on 31 July 2007 all marked by green dotted lines in Figs. 3 and 4 corresponded to shock crossings with the presence of a sharp single directional jump in the magnetic field and the generation of shock associated waves either upstream/downstream or both of the shock. Figures 5 and 6 provide a close-up view of all the above corresponding shocks. In the latest figures the vertical orange dotted lines represent examples of nonlinear structures that have not fully formed into a shock and the 

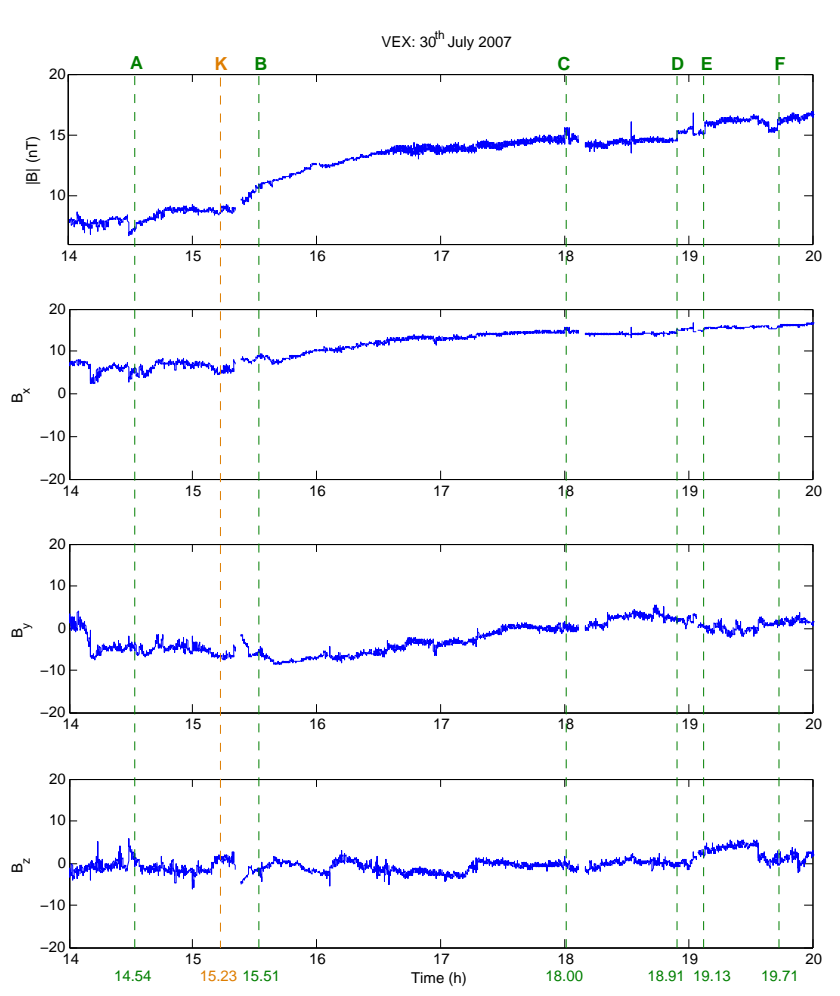

Fig. 5. Close-up view of the shocks presented in Fig. 3. Caption of Fig. 3 apply.

red dotted lines represent some examples of nonlinear structures that did not fulfil the criteria for a shock crossing. Table 1 summarises the properties of the above shocks, such as, shock crossing date, shock crossing time, magnetic field jump $(\Delta B)$, the ratio of magnetic field jump over magnetic field $(\Delta B / B)$, the angle between the magnetic field and the normal $\left(\theta_{B_{\mathrm{n}}}\right)$ and Mach number.

There are fairly noticeable similarities amongst the six shocks observed by VEX which are classified as weak shocks with relatively small values of $\Delta B / B(0.07,0.04,0.05,0.05$, 0.05 and 0.05$)$, field jump $\Delta B(0.51,0.38,0.71,0.72,0.72$ and 0.82$)$ and Mach number $(1.04,1.02,1.04,1.05,1.04$ and 1.05). The first three shocks (shock A, B and C) observed by VEX (presented in Table 1) are quasi-parallel with $\theta_{B_{\mathrm{n}}}<45^{\circ}\left(5^{\circ}, 28^{\circ}\right.$ and $36^{\circ}$ respectively). However, the final three shocks (shock D, E and F) are quasi-perpendicular with $\theta_{B_{\mathrm{n}}}>45^{\circ}\left(87^{\circ}, 63^{\circ}\right.$ and $\left.49^{\circ}\right)$. In general, the wave activity upstream and downstream of the shocks observed by VEX are insignificant, i.e., shock D shown in Fig. 7. In contrast, shock C illustrated in Fig. 8 possesses relatively significant wave activity both upstream and downstream of the shock.

On the other hand, the four shocks observed by ACE are rather different from those observed by VEX. The shocks observed by ACE are relatively stronger with larger values of $\Delta B / B(0.86,1.13,0.80$ and 0.25$)$, field jump $\Delta B(1.14$, $1.77,1.29$ and 0.79$)$ and Mach number $(1.84,2.04,1.76$ and 1.25) correspondingly. Three of these shocks (shocks
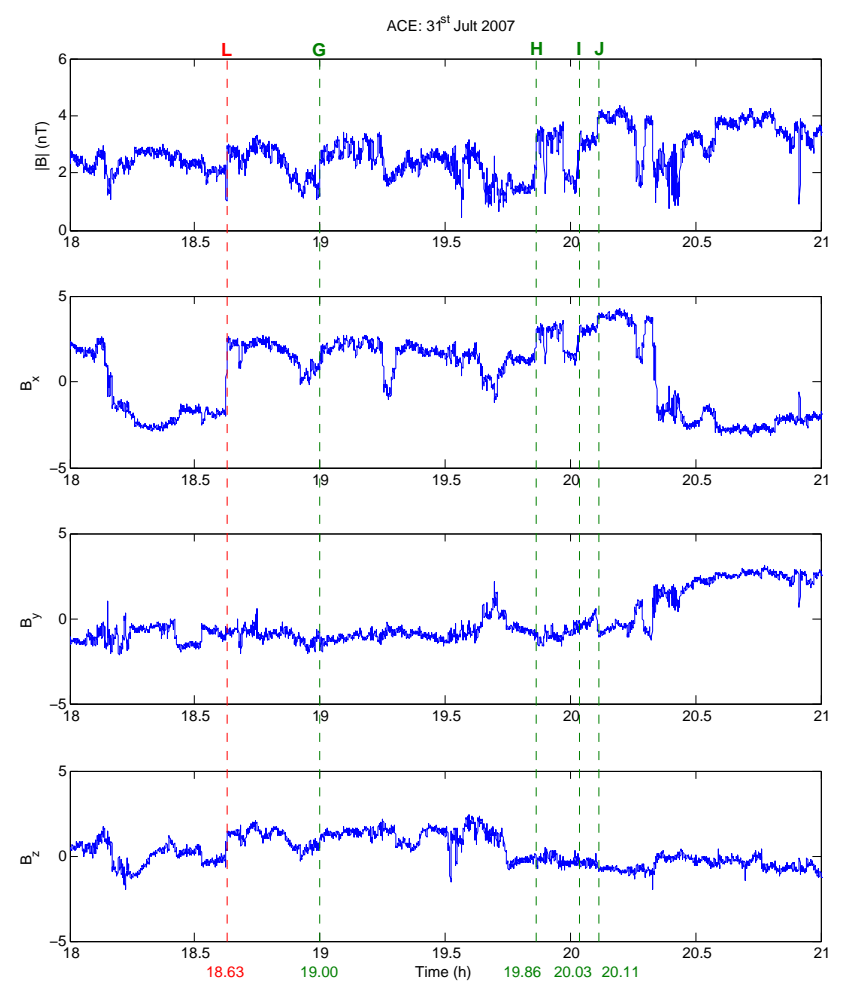

Fig. 6. Close-up view of the shocks presented in Fig. 4. Caption of Fig. 4 apply.

$\mathrm{G}, \mathrm{H}$ and I given in Table 1) are quasi-perpendicular with $\theta_{B_{\mathrm{n}}}>45^{\circ}\left(85^{\circ}, 69^{\circ}\right.$ and $55^{\circ}$ respectively). Nevertheless, the final shock (shock J) observed by ACE is quasi-parallel with $\theta_{B_{\mathrm{n}}}<45^{\circ}\left(34^{\circ}\right)$. In general, the wave activity upstream and downstream of the shocks observed by ACE are insignificant. However, shock H presented in Fig. 9 possesses relatively significant wave activity downstream of the shock. Also there appears to be a significant structure at the foot of shock I illustrated in Fig. 10 that may have formed as a result of collision between two shocks. Other nonlinear structures in the magnetic field measurements observed by VEX and ACE did not fulfil the criteria for a shock crossing. For example, Fig. 11 illustrates a nonlinear structure observed by ACE at 18.60 UTC on 31 July 2007 that did not fulfil the criteria for a shock crossing. In this case, the main magnetic field component $\left(B_{L}\right)$ crosses the zero axis in the shock coordinate frame.

The observation of fewer but stronger shocks by ACE compared to VEX provides persuasive indication of shock strengthening by coalescence as it was reported by Russell et al. (2009) for shocks associated with CIRs.

In this study both the coplanarity and minimum variance methods were used to calculate the field component along the normal direction, the component in the shock plane parallel to the projection of the upstream magnetic field and the component in the plane of the shock perpendicular to the 
Table 1. Summary of shock properties observed at VEX and ACE during the leading CME crossing. Shocks A, B, C, D, E and F were observed by VEX on 30 July 2007 whereas shocks G, H, I, J were observed by ACE on 31 July 2007. The columns of the table left to right represent shock crossing date, shock crossing time, magnetic field jump $(\Delta B)$, the ratio of magnetic field jump over magnetic field $(\Delta B / B)$, the angle between the magnetic field and the normal $\left(\theta_{B_{\mathrm{n}}}\right)$ and Mach number.

\begin{tabular}{lclrrrrr}
\hline Spacecraft & Shock & Date & Time (h) & $\delta B(\mathrm{nT})$ & $\delta B / B$ & $\theta_{B_{\mathrm{n}}}^{\circ}$ & Mach No. \\
\hline VEX & A & 30 July 2007 & 14.54 & 0.51 & 0.07 & 5 & 1.04 \\
VEX & B & 30 July 2007 & 15.51 & 0.38 & 0.04 & 28 & 1.02 \\
VEX & C & 30 July 2007 & 18.00 & 0.71 & 0.05 & 36 & 1.04 \\
VEX & D & 30 July 2007 & 18.91 & 0.72 & 0.05 & 87 & 1.05 \\
VEX & E & 30 July 2007 & 19.13 & 0.72 & 0.05 & 63 & 1.04 \\
VEX & F & 30 July 2007 & 19.71 & 0.82 & 0.05 & 49 & 1.05 \\
\hline ACE & G & 31 July 2007 & 19.00 & 1.14 & 0.86 & 85 & 1.84 \\
ACE & H & 31 July 2007 & 19.86 & 1.77 & 1.13 & 69 & 2.04 \\
ACE & I & 31 July 2007 & 20.03 & 1.29 & 0.8 & 55 & 1.76 \\
ACE & $\mathrm{J}$ & 31 July 2007 & 20.11 & 0.79 & 0.25 & 34 & 1.25 \\
\hline
\end{tabular}
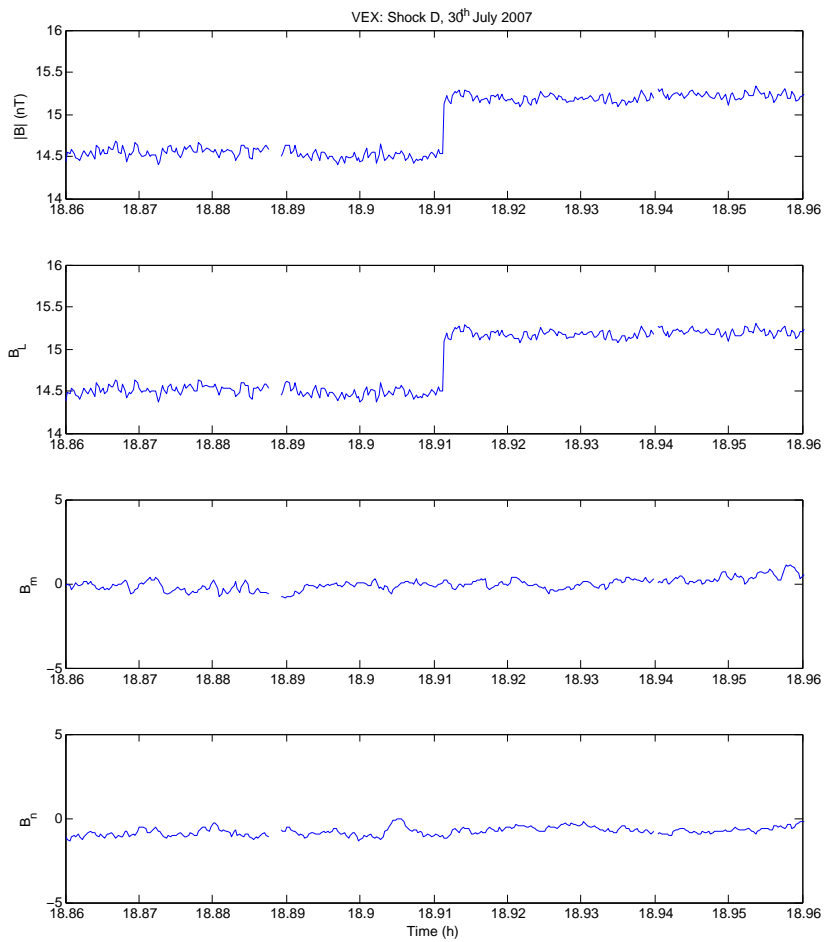

Fig. 7. Shock D: observed by VEX at 18.91 UTC on 30 July 2007. $|B|, B_{L}, B_{\mathrm{m}}$ and $B_{\mathrm{n}}$ are the magnetic field magnitude, the component in the shock plane parallel to the projection of the upstream magnetic field, the component in the plane of the shock perpendicular to the projection of the upstream field and the field component along the normal direction respectively.

projection of the upstream field. The Mach number was estimated using Eq. (1), the same formula as of Balikhin et al. (2008) where $M$ represents Mach number, $B_{\mathrm{d}}$ and $B_{\mathrm{u}}$ are upstream and downstream magnetic field measurements respectively, and $\theta_{B_{\mathrm{n}}}$ is the angle between the magnetic field
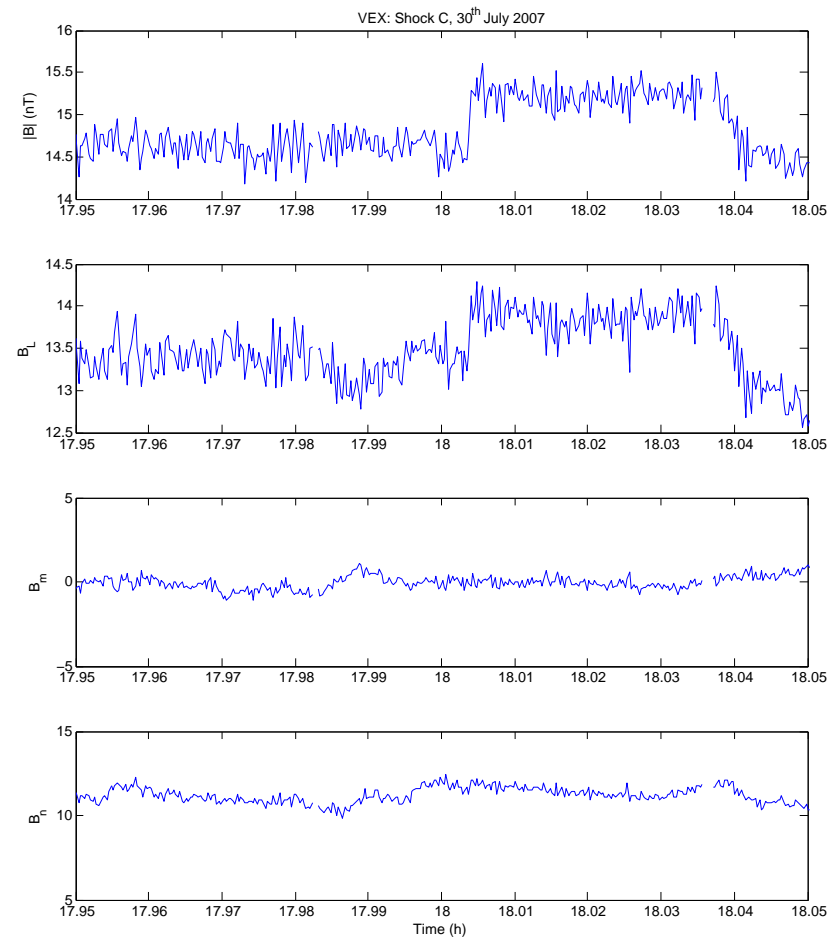

Fig. 8. Shock C: observed by VEX at 18.00 UTC on 30 July 2007. See caption of Fig. 7 for $|B|, B_{L}, B_{\mathrm{m}}$ and $B_{\mathrm{n}}$.

and the normal.

$M-1 \approx\left(\frac{B_{\mathrm{d}}}{B_{\mathrm{u}}}-1\right) \sin ^{2} \theta_{B_{\mathrm{n}}}$

The ENLIL simulations reveal that the trailing CME arrived at VEX as displayed in Fig. 12 at approximately 02:00 on 1 July 2007 when the VEX spacecraft was within the Venusian bow shock. Hence, during this period there is a significant gap in the clean magnetic field data recorded by VEX. Consequently, it would not be possible to identify any 

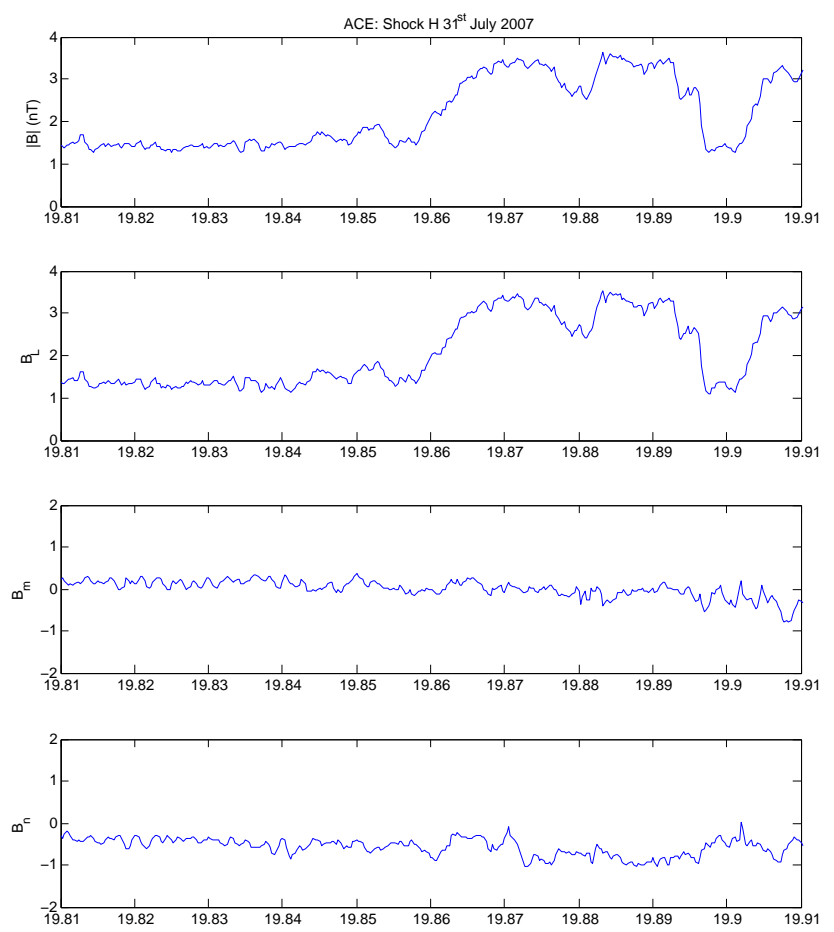

Fig. 9. Shock H: observed by ACE at 19.86 UTC on 31 July 2007. See caption of Fig. 7 for $|B|, B_{L}, B_{\mathrm{m}}$ and $B_{\mathrm{n}}$.
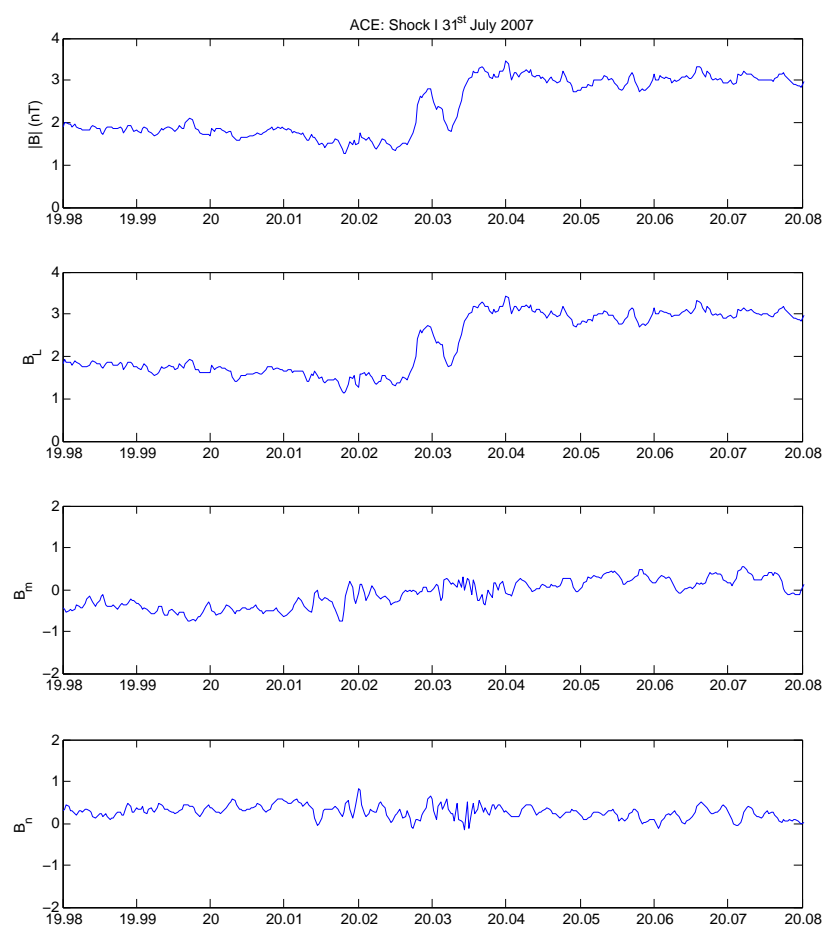

Fig. 10. Shock I: observed by ACE at 20.03 UTC on 31 July 2007. See caption of Fig. 7 for $|B|, B_{L}, B_{\mathrm{m}}$ and $B_{\mathrm{n}}$.
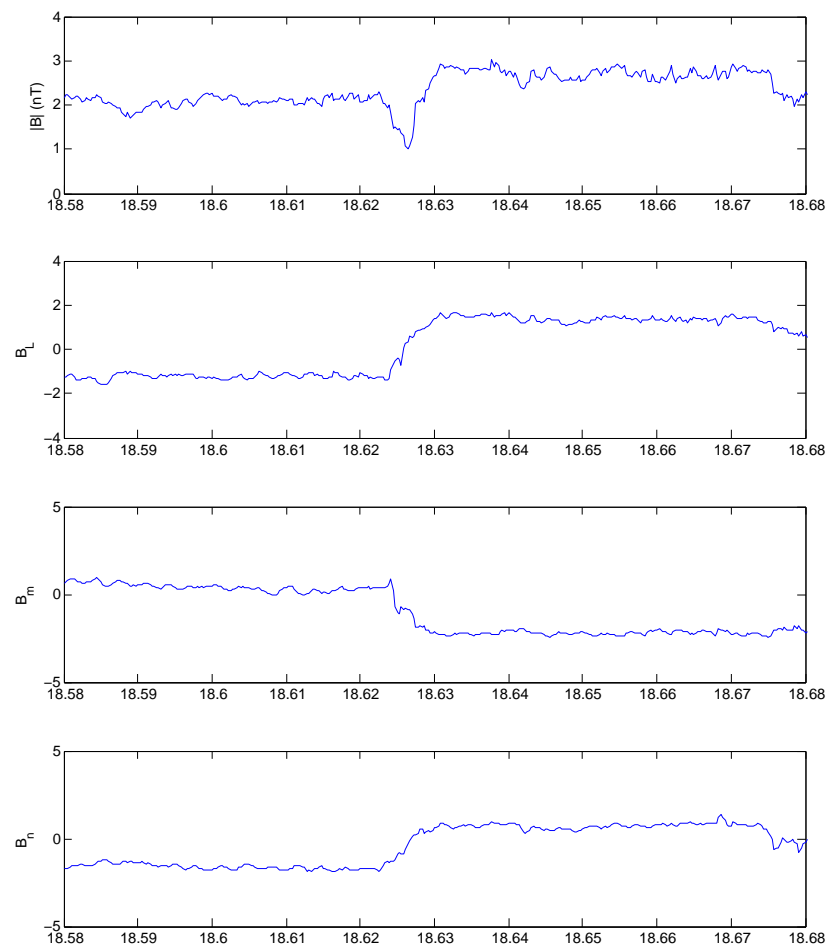

Fig. 11. An example of a nonlinear structure that did not fulfil criteria for a shock crossing. This structure was observed by ACE at 20.11 UTC on 31 July 2007. In this case, the main magnetic field component $\left(B_{L}\right)$ crosses the zero axis in the shock coordinated frame. See caption of Fig. 7 for $|B|, B_{L}, B_{\mathrm{m}}$ and $B_{\mathrm{n}}$.

CME associated shock crossings that may have occurred during this data gap. For this reason the trailing CME was not studied further.

For validation purposes, the magnetic field data measurements recorded by WIND were also analysed during the leading CME crossing on 31 July 2007. It was found that WIND, similar to ACE, also observed a total of four relatively strong shocks associated with the leading CME. This reiterates that stronger shocks may have formed as a result of coalescence of weaker shocks.

\section{Discussion and conclusions}

In this study it was found that there were a total of six shocks observed by VEX and only four but stronger shocks observed by ACE associated with the crossing of a partial halo CME on 30 July 2007 at the Venusian orbit and a day later at the Earth orbit . This indicates that the shocks observed at ACE may have strengthened by coalescence of weaker shocks observed earlier at VEX, and more importantly the formation of stronger shocks resulting from this coalescence takes place between Venus and the Earth. It is the balance of nonlinear steepening and some counterbalancing processes such as dispersion and resistivity that leads to the formation of shocks. 


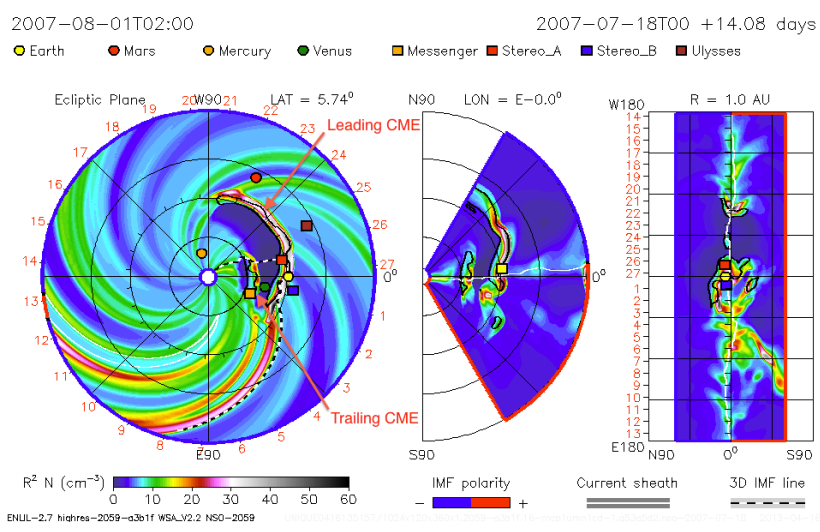

Fig. 12. The CCMC's ENLIL with a cone model simulation results. The estimated arrival time of the leading partial halo CME at Venus is approximately 02:00 on 1 August 2007 using the above simulation results. The red arrows mark the leading and trailing CMEs.

The majority of subcritical shocks observed in the vicinity of the Earth are fast magnetosonic dispersive shocks. These shocks are formed when the dispersion of fast magnetosonic waves counterbalances the nonlinear steepening (Kennel, 1985). The steepening of the nonlinear structure can be analytically described as the transfer of energy to the shorter scales. If the MHD approximation is valid then the waves on these new shorter scales will propagate with the same speed as the initial wave due to the non-dispersive characteristics of the MHD waves. Continuous steepening will pump more energy into these scales leading to an increase in the amplitude of the corresponding waves, and at some stage the later waves will also become subjected to nonlinear steepening where the energy will be transferred into even shorter scales and so on. Eventually this cascade will stop at extremely short scales where the MHD approximation will be invalid for their description. At this point it would be necessary to use the two fluid MHD model. The small-amplitude waves described by two fluid MHD are dispersive (Kennel, 1985), i.e. their phase velocity depends on the spatial scale. Waves with short scale will either propagate upstream relative to the front and form a wave precursor in case of quasi-perpendicular geometry or in almost perpendicular case when $\cos \left(\theta_{B_{\mathrm{n}}}\right)<\sqrt{\mu}$ they will be convected downstream and form wave train. Here $\mu$ is the ratio of electron and ion masses. The spatial scales of such fast magnetosonic shocks are determined by the whistler dispersion scale $\cos \left(\theta_{B_{\mathrm{n}}}\right)\left(c / \omega_{\mathrm{pi}}\right)$, where $\omega_{\mathrm{pi}}$ is the ion plasma frequency (Krasnoselskhikh et al., 2013). As the nonlinear structures associated with the CME steepens, the time when they reach local whistler dispersive scale depends upon the local plasma parameters that vary due to the non-uniformity of the CME itself. At that stage local dispersive shocks will be formed in various locations. Generally the propagation velocity of these shocks should differ as the plasma parameters are not uniform, leading to the coalescence in at least some of them. Such shocks can be effective accelerators of ions due to multiple reflections from their colliding fronts. This process of multiple shock formation is beyond the classical MHD approach, as the MHD does not account for wave dispersion.

In future this work can be extended to include CME associated shocks during solar wind conjunction periods between VEX and other spacecrafts near the Earth orbit such as the STEREO (Solar TErrestrial RElations Observatory) satellites. In this case, more CME associated shocks can be studied in order to understand further the formation of stronger shocks resulting from the coalescence of weaker shocks between the orbits of Venus and the Earth.

Acknowledgements. Simulation results have been provided by the Community Coordinated Modelling Center at Goddard Space Flight Center through their public Runs on Request system (http: //ccmc.gsfc.nasa.gov). The CCMC is a multi-agency partnership between NASA, AFMC, AFOSR, AFRL, AFWA, NOAA, NSF and ONR. The ENLIL with Cone Model Model was developed by the D. Odstrcil at the University of Colorado at Boulder. The authors would like to acknowledge discussions with C. T. Russell, A. P. Dimmock, S. N. Walker and S. A. Pope.

Topical Editor M. Gedalin thanks two anonymous referees for their help in evaluating this paper.

\section{References}

Balikhin, M. A., Zhang, T. L., Gedalin, M., Ganushkina, N. Y., and Pope, S. A.: Venus Express observes a new type of shock with pure kinematic relaxation. Geophys. Res. Lett. 35, 1103-1107, doi:10.1029/2007GL032495, 2008.

Falkenberg, T. V., Vrsnal, B., Taktakishvili, A., Odstrcil, D., MacNeice, P., and Hesse, M.: Investigations of the sensitivity of a coronal mass ejection model (ENLIL) to solar input parameters, J. Space Weather., 8, 6004-6014, doi:10.1029/2009SW000555, 2010.

Falkenberg, T. V., Vennerstrom, S., Brain, D. A., Delory, G., and Taktakishvili, A.: Multipoint observations of coronal mass ejection and solar energetic particle events on Mars and Earth during November 2001, J. Geophys. Res., 116, 6104-6118, doi:10.1029/2010JA016279, 2011.

Gopalswamy, N., Lara, A., Lepping, R. P., Kaiser, M. L., Berdichevsky, D., and Cyr, O. C. St.: Interplanetary acceleration of coronal mass ejections, J. Res. Lett., 27, 145-148, 2000.

Gopalswamy, N., Lara, A., Manoharan, P. K., and Howard, R. A.: An empirical model to predict the 1-AU arrival of interplanetary shocks, Adv. Space Res., 36, 2289-2294, 2005.

Gosling, J. T., Bame, S. J., Feldman, W. C., McComas, D. J., Phillips, J. L., Goldstein, B. E., and Neugebauer, M.: Latitudinal variation of solar wind corotating stream interaction regions: ULYSSES, Geophys. Res. Lett. 21, 2789-2792, doi:10.1029/93GL03116, 1993.

Hundhausen, A.: Coronal Mass Ejections. The many faces of the sun: a summary of the results from NASA's Solar Maximum Mission, edited by: Strong, K. T., Saba, J. L. R., Haisch, B. M., and Schmelz, J. T., Springer: New York, 143-200, 1999. 
Jian, L., Russell, C. T., Luhmann, J. G., and Skoug, R. M.: Properties of Interplanetary Coronal Mass Ejections at One AU During 1995-2004, Solphys., 239, 393-436, 2006.

Kahler, S., Pizzo, V. J., Holzer, T. E., and Sime, D. G.: Proceedings of the Sixth International Solar Wind Conference, Tech. Note NCAR/TN-306+Proc, 181, Natl. Cent. Atmos. Res., Boulder, CO, 215, 1988.

Kennel, C. F.: A quarter century of collisionless shock research, in Collisionless Shocks in the Heliosphere: A Tutorial Review, edited by: Stone, R. G. and Tsurutani, B. T., Geophysical Monograph: American Geophysical Union, Washington, 34, 1-36, 1985.

Krasnoselskikh, V., Balikhin, M., Walker, S. N., Schwartz, S., Sundkvist, D., Lobzin, V., Gedalin, M., Bale, S. D., Mozer, F., Soucek, J., Hobara, Y., and Comisel, H.: The Dynamic Quasiperpendicular Shock: Cluster Discoveries, Space Sci. Rev., 178, 535-598, 2013.

Odstrcil, D., Riley, P., and Zhao, X. P.: Numerical simulation of the 12 May 1997 interplanetary CME event, J. Geophys. Res., 109, 2116-2123, doi:10.1029/2003JA010135, 2004.

Russell, C. T., Jian, L. K., Blanco Cano, X., Luhmann, J. G., and Zhang, T. L.: STEREO observations of shock formation in the solar wind, Geophys. Res. Lett., 36, 2103-2105, doi:10.1029/2008GL036337, 2009.
Stone, E. C., Frandsen, A. M., Mewaldt, R. A., Christian, E. R., Margolies, D., Ormes, J. F., and Snow, F.: The Advanced Composition Explorer, Space Sci. Rev., 86, 1-22, 1998.

Taktakishvili, A., Kuznetsova, M., MacNeice, P., Hesse, M., Rastatter, L., Pulkkinen, A., Chulaki, A., and Odstrcil, D.: Validation of the coronal mass ejection predictions at the Earth orbit estimated by ENLIL heliosphere cone model, J. Space Weather, 7, 3004 3010, doi:10.1029/2008SW000448, 2009.

Zhang, T. L., Baumjohann, W., Delva, M., Auster, H. U., Balogh, A., Russell, C. T., Barabash, S., Balikhin, M., Berghofer, G., Biernat, H. K., Lammer, H., Lichtenegger, H., Magnes, W., Nakamura, R., Penz, T., Schwingenschuh, K., Voros, Z., Zambelli, W., Fornacon, K. H., Glassmeier, K. H., Richter, I., Carr, C., Kudela, K., Shi, J. K., Zhao, H., Motschmann, U., Lebreton, J. P.: Magnetic field investigation of the Venus plasma environment: Expected new results from Venus Express, Planet. Space Sci., 54, 1336-1343, 2006. 